\title{
Are the Recommended Criteria for Clinically Insignificant Prostate Cancer Applicable to 12-core Prostate Biopsy Scheme? A Multicentre Study of Urooncology Association, Turkey
} \author{
Members of Urooncology Association \\ 1/zmir Bozyaka Training and Research Hospital, Clinic of Urology, Izmir, Turkey \\ 2Ege University Faculty of Medicine, Department of Urology, Izmir, Turkey \\ ${ }^{3}$ Dokuz Eylül University Faculty of Medicine, Department of Pathology, Izmir, Turkey \\ 4 Hacettepe University Faculty of Medicine, Department of Urology, Ankara, Turkey \\ ${ }^{5}$ Çukurova University Faculty of Medicine, Department of Urology, Adana, Turkey \\ ${ }^{6}$ Dokuz Eylül University Faculty of Medicine, Department of Urology, Izmir, Turkey \\ ${ }^{7}$ Gazi University Faculty of Medicine, Department of Urology, Ankara, Turkey \\ ${ }^{8}$ Ankara University Faculty of Medicine, Department of Urology, Ankara, Turkey \\ ${ }^{9}$ Celal Bayar University Faculty of Medicine, Department of Urology, Manisa, Turkey \\ 10 Mehmet Ali Aydınlar University Kadıköy Hospital, Clinic of Urology, Istanbul, Turkey \\ ${ }^{11}$ Mehmet Ali Aydınlar University Altunizade Hospital, Clinic of Urology, Istanbul, Turkey
}

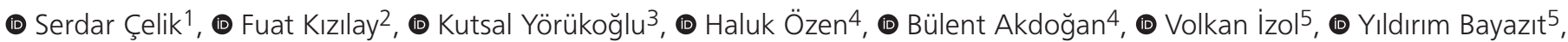

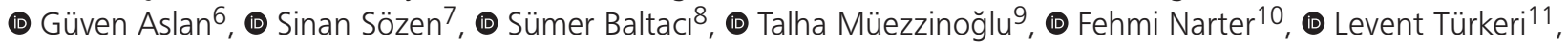

\begin{abstract}
Objective: The aim of this study is to investigate the relevance of the Epstein criteria for the 12-core transrectal prostate biopsy (TRUS-Bx) scheme with the evaluation of clinicopathologic data recorded in the Urologic Cancer Database - Prostate (UroCaD-P), Urooncology Association, Turkey (UOAT).

Materials and Methods: Patients with detailed pathological 12-core TRUS-Bx data for each biopsy core and who underwent RP due to PCa were included in this study. A total of 1167 patients from seven different centres were analysed. TRUS-Bx pathological findings were separately evaluated in the areas matching the sextant biopsy (6-core paramedian-lateral) scheme and in all 12-core biopsy areas (12-core biopsy scheme). Overall detection rates of PCa and ratios of clinically significant (sPCa) and insignificant PCa (insPCa) after RP were defined and compared between the biopsy schemes. Biopsy findings, according to the Epstein criteria, were also compared between the two schemes. A model for each biopsy scheme was created, including the Epstein criteria and additional biopsy findings using logistic regression analysis to predict clinically SPCa after RP.

Results: There was a high correlation for the prediction of clinically insPCa between the two biopsy schemes in the same population. However, 7.3\% of PCa could not be diagnosed in the 6-core TRUS-Bx scheme. Also, $69.4 \%$ of these had clinically SPCa according to the Epstein criteria in 12-core TRUS-Bx scheme and $51.8 \%$ of these were clinically sPCa after RP. The presence of perineural invasion (PNI) in 12-core biopsy was also significant regarding predicting $s \mathrm{PCa}$ ( $\mathrm{p}<0.001$ ).

Conclusion: The Epstein criteria in 12-core prostate biopsy provide a better prediction of clinically sPCa than the 6-core biopsy scheme. Biopsy PNI findings appeared to improve the effectiveness of 12-core prostate biopsy, in addition to the Epstein criteria.

Keywords: Prostate cancer, radical prostatectomy, clinically insignificant prostate cancer, Epstein criteria, 12-core prostate biopsy scheme
\end{abstract}

\section{Introduction}

Prostate cancer (PCa) is the most common cancer in men (1). Currently, diagnosis is via TRUS-guided biopsy (TRUS-Bx) based on prostate-specific antigen (PSA) level and digital rectal examination. However, not all forms of PCa will progress, and detection of clinically insignificant $\mathrm{PCa}$ (insPCa) may cause over-treatment in some patients. Although active surveillance

Cite this article as: Çelik S, Kızılay F, Yörükoğlu K, Özen H, Akdoğan B, İzol V, Bayazıt Y, Aslan G, Sözen S, Baltacı S, Müezzinoğlu T, Narter F, Türkeri L. Are the Recommended Criteria for Clinically Insignificant Prostate Cancer Applicable to 12-Core Prostate Biopsy Scheme? A Multicentre Study of Urooncology Association, Turkey. Bull Urooncol 2021;20(1):19-25

Address for Correspondence: Serdar Çelik, İzmir Bozyaka Training and Research Hospital, Clinic of Urology, İzmir, Turkey Phone: +90 5057019631 E-mail: serdarcelik84@hotmail.com ORCID-ID: orcid.org/0000-0003-0939-9989

Received: 09.03.2020 Accepted: 25.03.2020 
is an increasingly adopted management approach preventing unnecessary treatment in this patient category (very low-risk localised PCa), a substantial number of patients are still subjected to surgical or radiation-based interventions (2). Therefore, accurate identification of the clinical significance of tumours is crucial in avoiding unnecessary treatment with potential side effects or delay of curative therapy for whom it is required.

Initial attempts for a valid definition of clinical significance were based on tumour volume in 1993 by Stamey et al. (3). Later, preoperative criteria for the prediction of insPCa were defined in 1994 by Epstein et al. (4). Based on sextant biopsy findings, it was defined as a tumour $<0.2 \mathrm{~mL}$, organ-confined disease and a Gleason score (GS) $<7$. This comprised $16 \%$ of all $\mathrm{PCa}$ in their series (4). The initial report's positive and negative predictive values were $95 \%$ and $66 \%$, respectively, for insPCa (4). Subsequently, the Epstein criteria began to be used to predict insPCa to categorise patients for surveillance. However, diverse concordance ratios (37\%-96.9\%) were reported for the predictive ability of the Epstein criteria in various studies over time (5). During the same period, optimisation studies of TRUS biopsy schemes resulted in a general acceptance of obtaining 12 cores for biopsy. Although the Epstein criteria are assumed to be valid for 12-core biopsies, very scarce information is available in the current literature to support this view.

Therefore, we investigated the validity of the Epstein criteria, as defined according to the sextant biopsy scheme, for the currently utilised 12-core prostate biopsy protocol by analysing the clinicopathologic data recorded in the Urologic Cancer Database - Prostate (UroCaD-P), Urooncology Association, Turkey (UOAT).

\section{Materials and Methods}

In this study, we retrospectively reviewed the data of 3,300 patients in the UroCaD-P, UOAT between 2007 and 2019. Data were anonymised entirely in compliance with the local regulations at the source centre before being recorded in the UroCaD-P. Patients who had detailed 12-core TRUS-Bx pathologic data for each biopsy core and subsequently underwent radical prostatectomy (RP) (open, laparoscopic or robotic) due to PCa were included in the study. Patients with incomplete data for TRUS-Bx and/or RP were excluded. As a result, 1,167 patients from 7 different centres were evaluated in the study. Pathological findings were separately evaluated in the areas matching the sextant biopsy (6 cores paramedian-lateral) scheme and all 12 -core biopsy areas (6 cores paramedian-lateral and 6 cores far-lateral) and were separately entered into the database for each patient. Detection rates of $\mathrm{PCa}$ and ratios of clinically significant (sPCa) and insPCa after RP were separately evaluated and compared between the biopsy schemes. Prediction levels of clinically insPCa were defined according to the Epstein criteria. Also, true clinically insPCa was defined according to the final pathology report after RP (organ-confined PCa and no GS 4 or 5) (4). Proportions of patients who met the Epstein criteria (clinical stage T1c, PSA density $\leq 0.15 \mathrm{ng} / \mathrm{mL} / \mathrm{cm}^{3}, \leq 6 \mathrm{GS}$ (or Gleason grade group 1), $\leq 2$ positive biopsy cores and $\leq 50 \%$ percentage of tumour in positive biopsy core) were compared between the biopsy schemes. Accordingly, GS (according to 2005 modified
Gleason grading system), the number of positive cores and percentage of tumour in positive cores were compared between the sextant and 12-core TRUS-Bx schemes. In addition, PSA, PSA density, age and RP pathological findings of all patients were evaluated.

Two different models were created for each biopsy scheme based on the Epstein criteria alone and additional biopsy findings to predict the clinical significance of the tumours after RP.

\section{Statistical Analysis}

The non-parametric paired Wilcoxon test and chi-square test were used to analyse the relationships between categorical and independent variables. Also, the chi-square test, McNamer test and correlation analysis were used for the analysis of categorical variables and $p$-value, estimated risks (OR), Kappa score, Pearson's R correlation coefficient (R) and confidence intervals $(\mathrm{Cl})$, positive predictive values (PPV) and accuracy rates were given. The ability of the two different biopsy schemes to predict clinically insPCa after RP was evaluated using a logistic regression model. The Statistical Package for the Social Sciences version 22.0 was used for all statistical analysis. P-values less than 0.05 were considered statistically significant.

\section{Results}

A total of 1,167 patients with a median age of 63 years and a PSA level of $7.5 \mathrm{ng} / \mathrm{mL}$ were investigated in the study. The patients' demographic data, 12-core biopsy pathologic data and RP pathologic data are given in Table 1. Among patients, 767 (65.7\%) had clinically sPCa, and 400 (34.3\%) had clinically insPCa after RP. According to the prediction of the Epstein criteria, there were 143 patients with clinically insPCa after the evaluation of the 6-core TRUS-Bx scheme. In contrast, there were 111 patients, according to the 12-core TRUS-Bx scheme (Table 2). In evaluating 143 clinically insPCa patients who were predicted with the 6-core TRUS-Bx scheme, 33 of these patients were predicted as clinically $\mathrm{SPCa}$ according to the 12-core TRUS-Bx scheme. In addition, although PCa was diagnosed in the 12-core TRUS-Bx scheme, 85 (7.3\%) patients had no cancer according to the 6-core TRUS-Bx scheme. Also, 59 (69.4\%) of these 85 patients were predicted as clinically sPCa according to the Epstein criteria in the 12-core TRUS-Bx scheme, and 44 (51.8\%) of them were found to have clinically sPCa after RP. The results of predicting clinically $\mathrm{SPCa}$ and insPCa according to the Epstein criteria and analysis of additional pathological findings in the 6- and 12-core TRUS-Bx schemes are given in Table 3. The sensitivity, specificity, PPV and negative predictive values (NPV) of the sextant TRUS-Bx scheme for true clinically sPCa after RP was $94.9 \%, 26 \%, 71.1 \%$ and $72.7 \%$, respectively $(p<0.001$, OR: $6.559 \mathrm{Cl}: 4.43-9.71)$. The sensitivity, specificity, PPV and NPV of the 12-core TRUS-Bx scheme for the true clinically sPCa after RP were $97 \%, 22 \%, 70.5 \%$ and $79.3 \%$, respectively ( $p<0.001$, OR: $9,124 \mathrm{Cl}: 5,65-14,71)$. There was a high correlation between the two biopsy schemes $(p<0.001$; Pearson's R: 0.859). The model results for both 6-core and 12-core TRUS-Bx schemes according to the Epstein criteria and the model results of additional pathological findings added to the nomograms as predictive 
Table 1. Demographic data, 12-core biopsy pathologic data and RP pathologic data of the patients

Data, mean \pm standard deviation (minimum-maximum)

Age (years)

$\operatorname{BMl}\left(\mathrm{kg} / \mathrm{m}^{2}\right)$

PSA $(n g / m L)$

fPSA $(\mathrm{ng} / \mathrm{mL})$

$\mathrm{PV}\left(\mathrm{cm}^{3}\right)$

PSA density $\left(\mathrm{ng} / \mathrm{mL} / \mathrm{cm}^{3}\right)$

\begin{tabular}{|l|l|} 
& T1c \\
\cline { 2 - 2 } Clinical T stage, $n(\%)(n=1123)$ & T2a \\
\cline { 2 - 2 } & T2b \\
\cline { 2 - 2 } & T2c-T3 \\
\hline
\end{tabular}

GS of 12-core prostate biopsy

\begin{tabular}{|l|}
\hline 1 \\
\hline 2 \\
\hline 3 \\
\hline 4 \\
\hline 5 \\
\hline
\end{tabular}

PNI presence of 12-core prostate biopsy, $n$ (\%) $(n=1096)$

LVI presence of 12-core prostate biopsy, $n(\%)(n=1074)$

HGPIN presence of 12-core prostate biopsy, $n(\%)(n=1048)$

RP pathological T stage, $n(\%)(n=1166)$

GS of RP specimen

ISUP grade of RP specimen, $\mathrm{n}(\%)$

True clinically sPCa after RP, $\mathrm{n}(\%)$

True clinically insPCa after RP, $\mathrm{n}(\%)$

$\mathrm{n}=1167$

$62.7 \pm 6.5(42-86)$

$26.7 \pm 3.1(18.5-34.9)$

$10.5 \pm 11.5(1-125.7)$

$1.31 \pm 2.06(0.1-24.67)$

$52.1 \pm 27.2(14-200)$

$0.23 \pm 0.23(0.01-1.88)$

$265(22.7)$

$212(18.2)$

$37(3.2)$

$609(52.2)$

$6.65 \pm 0.83(4-10)$

$591(50.7)$

$334(28.6)$

$115(9.9)$

$68(5.8)$

$59(5.1)$

319 (29.1)

92 (8.6)

254 (24.2)

777 (66.6)

234 (20.1)

$151(12.9)$

$4(0.3)$

$6.81 \pm 0.85(4-10)$

437 (37.4)

437 (37.4)

148 (12.7)

65 (5.6)

$80(6.9)$

767 (65.7)

400 (34.3)

BMI: Body mass index, PSA: Prostate-specific antigen, fPSA: Free PSA, PV: Prostate volume, GS: Gleason score, ISUP: International society of urological pathology, PNI: Perineural invasion, LVI: Lymphovascular invasion, HGPIN: High grade prostatic intraepithelial hyperplasia, RP: Radical prostatectomy, sPCa: Significant prostate cancer, insPCa: Insignificant prostate cancer

\begin{tabular}{|c|c|c|c|c|}
\hline \multicolumn{2}{|l|}{ Biopsy results } & $\begin{array}{l}\text { 12-core TRUS-Bx scheme } \\
\text { group } \\
(\mathrm{n}=1167)\end{array}$ & $\begin{array}{l}\text { 6-core TRUS-Bx scheme } \\
\text { group } \\
(\mathrm{n}=1167)\end{array}$ & $\mathbf{p}^{*}$ \\
\hline \multirow{4}{*}{ Diagnosis, n (\%) } & Benign pathology & $0(0)$ & $85(7.3)$ & \multirow{4}{*}{ - } \\
\hline & $\mathrm{PCa}$ & $1167(100)$ & $1082(92.7)$ & \\
\hline & Clinically insPCa according to the Epstein criteria & $111(9.5)$ & $143(12.3)$ & \\
\hline & Clinically sPCa according to the Epstein criteria & $1056(90.5)$ & $1024(87.7)$ & \\
\hline \multicolumn{2}{|c|}{ Percentage of tumour in positive biopsy core } & $50.5 \pm 31.7(1-100)$ & $44.8 \pm 32.6(0-100)$ & $<0.001$ \\
\hline \multicolumn{2}{|c|}{ Number of positive biopsy core } & $3.34 \pm 2.45(1-12)$ & $2.32 \pm 1.6(0-6)$ & $<0.001$ \\
\hline
\end{tabular}


Table 3. Prediction of clinically sPCa and insPCa after RP according to the Epstein criteria and analysis of additional pathological findings in the 6- and 12-core biopsy schemes

\begin{tabular}{|c|c|c|c|c|}
\hline & $\begin{array}{l}\text { All patients } \\
(\mathrm{n}=1167)\end{array}$ & $\begin{array}{l}\text { Patients with true } \\
\text { clinically sPCa after RP } \\
\text { ( } n=767)\end{array}$ & $\begin{array}{l}\text { Patients with true } \\
\text { clinically insPCa after RP } \\
(\mathrm{n}=400)\end{array}$ & $\mathbf{p}^{*}$ \\
\hline \multicolumn{4}{|c|}{ Prediction of the Epstein criteria in 6-core TRUS-Bx scheme } & \multirow{3}{*}{$\begin{array}{l}\text { p<0.001 } \\
\text { OR: } 6.559 \text { (Cl: } 4.43-9.71) \\
\text { Pearson's R: } 0.303 \\
\text { Kappa: } 0.247 \\
\text { McNemar }<0.001\end{array}$} \\
\hline - Clinically insPCa, n (\%) & $143(12.3)$ & $39(5.1)$ & $104(26)$ & \\
\hline - Clinically sPCa, n (\%) & $1024(87.7)$ & $728(94.9)$ & $296(74)$ & \\
\hline \multicolumn{4}{|c|}{ Prediction of the Epstein criteria in 12-core TRUS-Bx scheme } & \multirow{3}{*}{$\begin{array}{l}\text { p<0.001 } \\
\text { OR: } 9.124 \text { (Cl: } 5.65-14.7) \\
\text { Pearson's R: } 0.307 \\
\text { Kappa: } 0.230 \\
\text { McNemar }<0.001\end{array}$} \\
\hline - Clinically insPCa, n (\%) & $111(9.5)$ & $23(3)$ & $88(22)$ & \\
\hline - Clinically sPCa, n (\%) & $1056(90.5)$ & $744(97)$ & $312(78)$ & \\
\hline PNI presence in 12-core biopsy, $n(\%)(n=1096)$ & $319(29.1)$ & $263(36.5)$ & $56(14.9)$ & $\begin{array}{l}\text { p }<0.001 \\
\text { OR: } 3.3 \text { (Cl:2.38-4.54) } \\
\text { Pearson's R: } 0.226\end{array}$ \\
\hline LVI presence in 12-core biopsy, $n(\%)(n=1074)$ & $92(8.6)$ & $79(11.2)$ & $13(3.5)$ & $\begin{array}{l}\mathrm{p}<0.001 \\
\text { OR: } 3.5 \text { (Cl: } 1.91-6.36) \\
\text { Pearson's R: } 0.131\end{array}$ \\
\hline $\begin{array}{l}\text { HGPIN presence in 12-core biopsy, } n(\%) \\
(n=1048)\end{array}$ & $254(24.2)$ & $175(25.7)$ & $13(21.5)$ & $\begin{array}{l}\mathrm{p}=0.133 \\
\text { OR: } 1.3 \text { (Cl: } 0.93-1.71) \\
\text { Pearson's R: } 0.046\end{array}$ \\
\hline
\end{tabular}

Table 4. In the same patients, created model results of predicting clinically SPCa and insPCa after RP according to the Epstein criteria and additional pathological findings in both 6- and 12-core biopsy schemes

\begin{tabular}{|c|c|c|c|}
\hline & p-value & $\operatorname{Exp}(B)$ & $\mathrm{Cl}$ \\
\hline \multicolumn{4}{|c|}{$\begin{array}{l}\text { Predictive model of the Epstein criteria in 6-core TRUS-Bx scheme } \\
\text { (Model } p<0.001 \text { ) }\end{array}$} \\
\hline - PSA & 0.383 & 1.055 & $0.935-1.191$ \\
\hline - PSA density & 0.008 & 0.383 & $0.188-0.777$ \\
\hline - Clinical T Stage & 0.028 & 0.234 & $0.064-0.856$ \\
\hline - Biopsy GS & $<0.001$ & 0.015 & $0.003-0.073$ \\
\hline - Tumour percentage of positive core & 0.014 & 0.346 & $0.149-0.804$ \\
\hline - Number of positive cores & 0.976 & 0.988 & $0.442-2.207$ \\
\hline \multicolumn{4}{|c|}{$\begin{array}{l}\text { Predictive model of the Epstein criteria in 12-core TRUS-Bx scheme } \\
\text { (Model } p<0.001 \text { ) }\end{array}$} \\
\hline - PSA & 0.413 & 1.052 & $0.932-1.118$ \\
\hline - PSA density & 0.013 & 0.401 & $0.196-0.822$ \\
\hline - Clinical T Stage & 0.032 & 0.238 & $0.064-0.884$ \\
\hline - Biopsy GS & $<0.001$ & 0.017 & $0.004-0.078$ \\
\hline - Tumour percentage of positive core & 0.002 & 0.259 & $0.110-0.612$ \\
\hline - Number of positive cores & 0.565 & 1.268 & $0.565-2.847$ \\
\hline \multicolumn{4}{|c|}{$\begin{array}{l}\text { New modelling of findings in } 12 \text { core prostate biopsy } \\
\text { (Model } p<0.001 \text { ) }\end{array}$} \\
\hline - Epstein criteria & $<0.001$ & 7.379 & $4.447-12.242$ \\
\hline - PNI presence in prostate biopsy & $<0.001$ & 2.514 & $1.771-3.568$ \\
\hline - LVI presence in prostate biopsy & 0.093 & 1.734 & 0.913-3.296 \\
\hline
\end{tabular}


factors for the sextant and 12-core TRUS-Bx scheme are given in Table 4. Analysis of data revealed the presence of perineural invasion (PNI) in the 12-core biopsy scheme as a significant predictor in both univariate and multivariate analyses in terms of sPCa ( $p<0.001$; OR: $3.3 \mathrm{Cl}$ : 2.38-4.54; Pearson's R: 0.226).

\section{Discussion}

The widespread use of PSA testing has led to over-diagnosis because of increased prostate biopsy rates and increased number of cores in each biopsy $(6,7)$. At the same time, over-treatment rate of RP also increased over time. After the published reports about RP series, $26-33 \%$ of RP specimens were clinically insPCa (organ-confined $\mathrm{PCa}$, tumour volume less than $0.2 \mathrm{cc}$, and no Gleason pattern 4 or 5$)(4,8)$. Our series found that $34.3 \%$ of patients had clinically insPCa after RP, consistent with the literature. Therefore, it is becoming more important to distinguish the clinically significant disease from clinically insPCa in the decision-making process before treatment to avoid unnecessary treatment interventions. Therefore, identification of insPCa for active surveillance became a major topic of interest. The Epstein criteria have been widely used for that purpose in clinical practice despite some deficiencies (9). Based on the final pathology results, predictive variables were suggested as $\leq 0.15 \mathrm{ng} / \mathrm{mL} / \mathrm{cm}^{3}$ PSA density, T1c clinical stage and favourable features on 6 -core prostate biopsy [ $\leq 6$ GS (Gleason grade group $1), \leq 2$ positive biopsy cores and $\leq 50 \%$ percentage of tumour in positive biopsy core] $(4,10)$.

When we look at each predictive factor evaluated in the Epstein criteria, PSA density was previously found to be useful to differentiate more aggressive PCa (11). It was also used as an inclusion criterion for AS $(12,13)$. Cut-off values of PSA density were defined as $0.15 \mathrm{ng} / \mathrm{mL} / \mathrm{cm}^{3}$ and $0.2 \mathrm{ng} / \mathrm{mL} / \mathrm{cm}^{3}$ in previous studies. In our evaluation and validation of the Epstein criteria with the 12-core biopsy scheme, the threshold of PSA density was taken at the level of $0.15 \mathrm{ng} / \mathrm{mL} / \mathrm{cm}^{3}$, like the original study, to predict clinically insPCa. The clinical stage T1c is a main factor for the Epstein criteria because it predicts about $30 \%$ of clinically insPCa after RP $(4,8)$.

One of the questions we aimed to answer is the optimal number and percentage of positive biopsy cores from a 12-core biopsy to predict a significant tumour at RP. In this context, some protocols recommend the threshold as the percentage of positive cores (14). In such protocols Dall'Era et al. (15) recommended the presence of $<6$ total GS, $<10 \mathrm{ng} / \mathrm{mL}$ PSA level, $\leq 33 \%$ positive cores and tumour presence in $\leq 50 \%$ of each positive core as indicators of insPCa. Similarly, van AS et al. (16), included clinical stage T1-2a, $\leq 7$ total GS $(3+4)$ or $\leq$ International Society of Urological Pathology grade $2,<15 \mathrm{ng} / \mathrm{mL}$ PSA level and $<50 \%$ positive biopsy cores. In summary, the primary purpose of all these criteria is to predict clinically insPCa and to avoid overtreatment in eligible patients. Many publications suggested that a low number of positive cores was associated with favourable pathological findings at RP specimens $(17,18,19)$. However, there are important studies questioning the role of a number of positive cores on biopsy as a predictive factor for insPCa $(18,19)$. In the current study, we found that the average number of positive biopsy cores was higher in the 12-core biopsy scheme than the 6-core biopsy scheme ( 3.34 vs $2.32, p<0.001)$. In the regression model for our population, the $\leq 2$ positive biopsy core finding was not a predictive factor for clinically insPCa in both 6- and 12-core biopsy schemes within the context of the Epstein criteria.

Presence of tumour in $<50 \%$ of the positive biopsy core was the best factor correlated with the prediction of insPCa among the Epstein criteria in the literature (17). In a recently published study, very low-risk patients ( $\leq 6 \mathrm{GS}, \leq 2$ positive biopsy core and $\leq 50 \%$ of tumour in positive core) and other low-risk patients ( $\leq 6 \mathrm{GS},>2$ positive core and/or $>50 \%$ percentage of tumour in positive core) were compared and a risk stratification, including tumour volume on biopsy was recommended for low-risk patients (20). In the current study, we found that the mean percentage of tumour in positive biopsy cores were higher in the 12-core biopsy scheme than in the 6-core biopsy scheme $(50.5 \%$ vs $44.8 \% ; p<0.001)$. When we look at the regression model in our study, the presence of $\leq 50 \%$ of tumour in positive biopsy core was an independent predictive factor for clinically insPCa in both 6- and 12-core biopsy schemes, consistent with the literature.

The current study aimed to evaluate the performance of the Epstein criteria for the 12-core prostate biopsy scheme. We also investigated the role of possible additional predictive factors that can be added to the criteria such as prostate biopsy PNI, lymphovascular invasion and others. In our cohort, the Epstein criteria in both 6-core and 12-core biopsy schemes significantly predicted clinically $\mathrm{sPCa}$ (or insPCa) and were found to correlate with each other. However, the 12-core biopsy scheme was superior for this prediction. However, despite the better performance of 12-core biopsy, only 88 of 400 (22\%) patients with true clinically insPCa at final pathology could be predicted. This finding indicates a major room for improvement. Thus, additional analysis of our data highlighted the presence of PNI at the biopsy specimen as a promising predictive factor. The finding of $\mathrm{PNI}$ in biopsy is shown as the extension of PCa cells along the nerve bundle in prostate tissue (21). It is reported in $20 \%$ of all biopsies harbouring PCa, which is generally accompanied by high GS and PSA levels (22).

Additionally, a high correlation level was shown between PNI on biopsy and extra prostatic extension and surgical margin positivity after RP $(22,23,24,25,26,27)$. However, PNI on biopsy was not always an independent predictive factor of sPCa $(28,29)$. Nevertheless, prostate biopsy PNI presence was an independent predictive factor for clinically SPCa at RP in our study when we incorporated this variable into the Epstein criteria.

In summary, there was a high correlation for the prediction of clinically SPCa/insPCa between the two biopsy schemes in the same patient population. Nevertheless, $7.3 \%$ of patients could not be diagnosed with PCa in 6-core TRUS-Bx scheme. Also, $69.4 \%$ of these patients (5.1\% of all) were clinically sPCa according to the Epstein criteria in the 12-core TRUS-Bx scheme, and $51.8 \%$ of them (3.8\% of all) were clinically sPCa after RP. According to our results, using the Epstein criteria with 12-core prostate biopsy provides better results in predicting clinically 
sPCa than 6-core biopsy. Furthermore, PNI on biopsy can be a useful predictive factor in addition to the Epstein criteria.

\section{Study Limitations}

The major limitations of our study are its retrospective nature and analysis. Therefore, indications for surgery were at the physician's discretion. Another important limitation is that there was no centralised pathological examination and the proposed changes in the Gleason grading system over time. However, multicentric pathological examinations by uropathologists at respective centres and long-term data acquisition may reflect a real-life nationwide picture.

\section{Conclusions}

The Epstein criteria in sextant prostate biopsy scheme predicted clinically significant PCa with high sensitivity in our cohort in concordance with the original publication and subsequent literature. The performance of biopsy the Epstein criteria in predicting insPCa at final pathology was better with 12-core prostate biopsy scheme in our cohort. In addition, incorporation of the biopsy PNI finding to the prediction model improved the performance of the Epstein criteria.

\section{Acknowledgements}

Publication: This study was presented in the $14^{\text {th }}$ Urooncology Winter Congress and Course on November 6-10 in 2019.

Contribution: There is not any contributors who may not be listed as authors.

Conflict of Interest: No conflict of interest was declared by the authors.

Financial Disclosure: The authors declared that this study received no financial support.

\section{Ethics}

Ethics Committee Approval: We investigated the validity of the Epstein criteria, as defined according to the sextant biopsy scheme, for the currently utilised 12-core prostate biopsy protocol by analysing the clinicopathologic data recorded in the Urologic Cancer Database - Prostate (UroCaD-P), Urooncology Association, Turkey (UOAT).

Informed Consent: Retrospective study.

Peer-review: Externally and internally peer reviewed.

\section{Authorship Contributions}

Concept: S.Ç., F.K., K.Y., F.N., L.T., Design: S.Ç., F.K., K.Y., F.N., L.T., Data Collection or Processing: H.Ö., B.A., V.I., Y.B., G.A., S.S., S.B., T.M., Analysis or Interpretation: S.Ç., K.Y., L.T., Literature Search: S.Ç., F.K., Writing: S.Ç., K.Y., L.T.

\section{References}

1. Siegel RL, Miller KD, Jemal A. Cancer statistics, 2019. CA Cancer J Clin 2019;69:7-34.

2. Mottet N, van den Bergh RCN, Briers E, et al. EAU Guideline on Prostate Cancer. 2019. Uroweb. Available: https://uroweb.org/ guideline/prostate-cancer/
3. Stamey T, Freiha F, McNeal J, et al. Localized prostate cancer. Relationship of tumour volume to clinical significance for treatment of prostate cancer. Cancer 1993;71(3 Suppl):933-938.

4. Epstein J, Walsh P, Carmichael M, Brendler CB. Pathologic and clinical findings to predict tumour extent of nonpalpable (stage T1c) prostate cancer. JAMA 1994;271:368-374.

5. Oon S, Watson R, O'Leary J, Fitzpatrick JM. Epstein Criteria for insignificant prostate cancer. BJU Int 2011;108:518-525.

6. Draisma G, Boer R, Otto SJ, et al. Lead times and overdetection due to prostate-specific antigen screening: estimates from the European Randomized Study of Screening for Prostate Cancer. J Natl Cancer Inst 2003;95:868-878.

7. Cooperberg MR, Lubeck DP, Mehta SS, et al. Time trends in clinical risk stratification for prostate cancer: implications for outcomes (data from CaPSURE). J Urol 2003;170(6 Pt 2):S21-S25; discussion S26-S27.

8. Carter HB, Sauvageot J, Walsh PC, Epstein JI. Prospective evaluation of men with stage T1C adenocarcinoma of the prostate. J Urol 1997;157:2206-2209.

9. Frankcombe DE, Li J, Cohen RJ. Redefining the Concept of Clinically Insignificant Prostate Cancer. Urology 2020;136:176-179.

10. Bastian PJ, Mangold LA, Epstein JI, Partin AW. Characteristics of insignificant clinical T1c prostate tumors. A contemporary analysis. Cancer 2004;101:2001-2005.

11. Kundu SD, Roehl KA, Yu X, et al. Prostate specific antigen density correlates with features of prostate cancer aggressiveness. I Urol 2007; 177:505-509.

12. Van den Bergh RC, Roemeling S, Roobol MJ, et al. Outcomes of men with screen-detected prostate cancer eligible for active surveillance who were managed expectantly. Eur Urol 2009;55:1-8.

13. Carter HB, Kettermann A, Warlick C, et al. Expectant management of prostate cancer with curative intent: an update of the Johns Hopkins experience. J Urol 2007;178:2359-2364; discussion 2364-2365.

14. Komisarenko $M$, Martin LJ, Finelli A. Active surveillance review: contemporary selection criteria, follow-up, compliance and outcomes. Transl Androl Urol 2018;7;243-255.

15. Dall'Era MA, Cooperberg MR, Chan JM, et al. Active surveillance for early-stage prostate cancer: review of the current literature. Cancer 2008;112:1650-1659.

16. van As NJ, Norman AR, Thomas K, et al. Predicting the probability of deferred radical treatment for localised prostate cancer managed by active surveillance. Eur Urol 2008;54:1297-1305.

17. Matoso A, Epstein JI. Defining clinically significant prostate cancer on the basis of pathological findings. Histopathology 2019;74:135-145.

18. Audenet F, Vertosick EA, Fine SW, et al. Biopsy core features are poor predictors of adverse pathology in men with grade group 1 prostate cancer. J Urol 2018;199;961-968.

19. Bokhorst LP, Valdagni R, Rannikko A, et al. A decade of active surveillance in the PRIAS study: an update and evaluation of the criteria used to recommend a switch to active treatment. Eur Urol 2016;70:954-960.

20. Tosoian JJ, Mamawala M, Patel HD, et al. Tumor volume on biopsy of low risk prostate cancer managed with active surveillance. J Urol 2018;199:954-960.

21. Hassan MO, Maksem J. The prostatic perineural space and its relation to tumor spread: an ultrastructural study. Am J Surg Pathol 1980;4:143-148.

22. DeLancey JO, Wood Jr DP, He C, et al. Evidence of perineural invasion on prostate biopsy specimen and survival after radical prostatectomy. Urology 2013;81:354-357.

23. Gutiérrez C, Terrasa F, Briones $G$, et al. Prognostic role of perineural invasion in prostate biopsy. Actas Urol Esp 2011;35:347-353.

24. Miller JS, Chen $\mathrm{Y}, \mathrm{Ye} \mathrm{H}$, et al. Extraprostatic extension of prostatic adenocarcinoma on needle core biopsy: report of 72 cases with clinical follow-up. BJU Int 2010;106:330-333. 
25. Celik S, Bozkurt O, Demir O, et al. Effects of perineural invasion in prostate needle biopsy on tumor grade and biochemical recurrence rates after radical prostatectomy. Kaohsiung J Med Sci 2018;34:385-390.

26. Bismar TA, Lewis JS Jr, Vollmer RT, Humphrey PA. Multiple measures of carcinoma extent versus perineural invasion in prostate needle biopsy tissue in prediction of pathologic stage in a screening population. Am J Surg Pathol 2003;27:432-440.
27. Harnden P, Shelley MD, Clements $H$, et al. The prognostic significance of perineural invasion in prostatic cancer biopsies: a systematic review. Cancer 2007;109:13-24.

28. Al-Hussain T, Carter HB, Epstein II. Significance of prostate adenocarcinoma perineural invasion on biopsy in patients who are otherwise candidates for active surveillance. J Urol 2011;186;470-473.

29. Trpkov C, Yilmaz A, Trpkov K. Perineural invasion in prostate cancer patients who are potential candidates for active surveillance: validation study. Urology 2014;84;149-152. 